\title{
Metástase linfonodal de adenoma pleomórfico recidivado - relato de caso
}

\section{Nodal metastasis of recurrent pleomorphic adenoma - case report}

Gabriel Silva Lima ${ }^{1}$. Jônatas Catunda de Freitas ${ }^{1,2}$. Francieudo Justino Rolim ${ }^{1,2}$. Glebert Monteiro Pereira ${ }^{1,2}$. Francisco Januário Farias Pereira Filho ${ }^{1,2}$. Luis Alberto Albano Ferreira ${ }^{1,2}$. Teresa Neuma Albuquerque Gomes Nogueira ${ }^{1}$.

1 Universidade Federal do Ceará (UFC), Fortaleza, Ceará, Brasil. 2 Hospital Universitário Walter Cantídio (HUWC), Fortaleza, Ceará, Brasil.

\section{RESUMO}

O adenoma pleomórfico é a neoplasia mais comum das glândulas salivares maiores e menores; entretanto, sua variante metastática é rara. Assim, o objetivo desse trabalho é relatar um caso dessa entidade. Paciente feminina de 42 anos realizou uma ressecção transoral de adenocarcinoma de glândulas salivares menores em mucosa jugal esquerda em 2008. Após 8 anos, apresentou recidiva tumoral e metástase para linfonodos submandibulares, sendo esta identificada como adenoma pleomórfico. Paciente foi submetida a ressecção da lesão, com maxilectomia e esvaziamento radical modificado, com reconstrução a partir de retalho do músculo masseter. Paciente foi encaminhada para radioterapia adjuvante e está livre de doença há 1 ano. A metástase dos adenomas pleomórficos se manifestam anos após o tumor primário. O mecanismo fisiopatológico da disseminação é incerto e marcadores tumorais de maior agressividade para esses adenomas estão sendo estudados. Reafirma-se, portanto, a necessidade de excisão completa e tecnicamente perfeita da neoplasia primária.

Palavras-chave: Adenoma pleomorfo. Recidiva local de neoplasia. Metástase linfática. Oncologia cirúrgica. Neoplasias de cabeça e pescoço.

\section{ABSTRACT}

Pleomorphic adenoma is the most common neoplasm of the major and minor salivary glands; however, its metastatic variant is rare. Thus, the purpose of this paper is to report a case of this entity. A 42-year-old female patient underwent a transoral resection of adenocarcinoma of minor salivary glands in left jugal mucosa in 2008. After 8 years, she presented tumor recurrence and metastasis to submandibular lymph nodes, which was identified as pleomorphic adenoma. The patient was submitted to resection of the lesion, with maxillectomy and modified radical emptying, with reconstruction from the masseter muscle flap. The patient was referred for adjuvant radiotherapy and has been free of disease for 1 year. The metastasis of pleomorphic adenomas manifests years after the primary tumor. The pathophysiological mechanism of dissemination is uncertain and tumor markers of greater aggressiveness for these adenomas are being studied. Therefore, the need for complete and technically perfect excision of the primary neoplasia is reaffirmed.

Keywords: Pleomorphic adenoma. Local Neoplasm Recurrence. Lymphatic Metastasis. Surgical Oncology. Head and Neck Neoplasms.

Autor correspondente: Gabriel Silva Lima, Rua Eugênio Gadelha, 315, Montese, Fortaleza, Ceará, Brasil. CEP: 60420-450. Telefone: +55 85 98573-5085. E-mail: gabrielsilvalima77@gmail.com

Conflito de interesses: Não há qualquer conflito de interesses por parte de qualquer um dos autores.

Recebido em: 01 Jul 2018; Revisado em: 19 Jan 2019; Aceito em: 14 Ago 2019. 


\section{INTRODUÇÃO}

O adenoma pleomórfico é a neoplasia mais comum das glândulas salivares maiores e menores. ${ }^{1}$ Apresenta crescimento lento, benigno e é composta por tecido epitelial e estromal. ${ }^{2}$ Por ser uma lesão benigna, espera-se que não dê metástases. Há, porém, uma entidade rara, chamada adenoma pleomórfico metastático (APM), que se apresenta histologicamente idêntica ao adenoma pleomórfico primário, em linfonodos, pulmões ou ossos. Sua mortalidade pode chegar a $22 \% .^{2}$

Relatamos o caso de uma paciente com adenocarcinoma de baixo grau recidivado, invadindo maxila com uma metástase linfonodal em nível Ib compatível com adenoma pleomórfico.

\section{RELATO DO CASO}

Paciente do sexo feminino de 42 anos realizou uma ressecção transoral de lesão submucosa bem delimitada de $3 \mathrm{~cm}$ em mucosa jugal esquerda em 2008, com diagnóstico pósoperatório de adenocarcinoma de glândulas salivares menores de baixo grau. Perdeu seguimento ambulatorial. Retornou apenas 8 anos após o tratamento inicial, apresentava recidiva volumosa e trismo. A lesão acometia mucosa jugal esquerda com extensão para rebordo alveolar superior da maxila, invadindo palato e placas pterigoides, além de apresentar grande componente insuflativo em área malar infiltrando pele e linfonodomegalia submandibular esquerda sugestiva de metástase (Figura 1). Biópsia incisional da área ulcerada em mucosa jugal teve como resultado adenoma pleomórfico. A paciente foi submetida à ressecção da lesão incluindo maxilectomia de inframesoestrutura esquerda ampliada para placas pterigoides e área de pele, associado ao esvaziamento radical modificado, e reconstrução com prótese obturatória confeccionada com retalho de $\mathrm{m}$. masseter para reconstruir mucosa jugal e fechamento primário da pele. (Figura 2).

A paciente evoluiu bem, com discreta perda do retalho de bochecha inferior e fístula salivar de rápida resolução. $\mathrm{O}$ resultado histopatológico mostrou ser um carcinoma ex-adenoma pleomórfico, margens livres, e no esvaziamento um linfonodo de 30 esvaziados, de $2 \mathrm{~cm}$, positivo para metástase de adenoma pleomórfico. A paciente foi encaminhada à radioterapia adjuvante e encontra-se bem, 12 meses após conclusão do tratamento sem evidência de doença.

Figura 1. A - lesão infiltrando pele e metástase linfonodal; B tomografia computadorizada (TC) mostrando invasão de maxilla; $\mathrm{C}$ - metástase linfonodal em nível Ib.

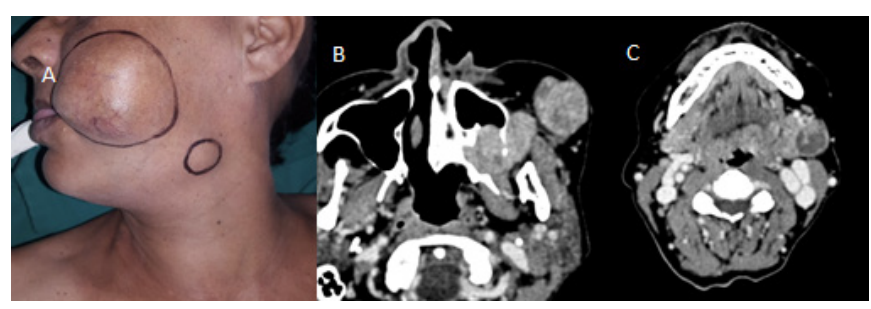

Figura 2. A - Incluído ilha de pele em conjunto da maxilectomia. B - lesão em mucosa de rebordo gengival superior. $\mathrm{C}$ - peça cirúrgica - retirada em monobloco. D - resultado tardio.

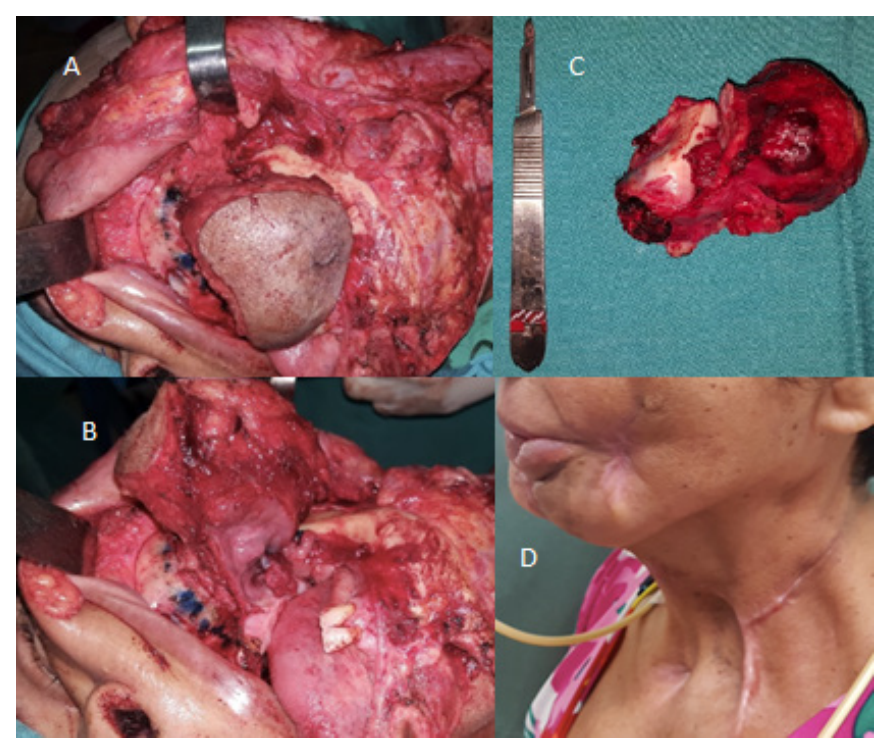

\section{DISCUSSÃO}

O adenoma pleomórfico é composto por uma matriz originada de tecido epitelial e mesenquimal, e tem comportamento benigno, embora possua potencial para malignização, originando o carcinoma ex-adenoma pleomórfico ou o carcinossarcoma.

Entretanto, desde 1940, uma rara entidade tem sido reconhecida, distinta das variantes malignas do adenoma previamente citadas, sendo chamada de adenoma pleomórfico metastático (APM). ${ }^{1}$ Sua peculiaridade reside no fato de - ao contrário do carcinoma ex-adenoma pleomórfico e do carcinossarcoma, que apresentam características de malignidade no tecido epitelial e/ou no estroma ${ }^{2}$ - manifestar-se como metástases histologicamente benignas.

Até a classificação de 2005 da OMS, o adenoma pleomórfico metastático era descrito como um tumor histologicamente igual ao adenoma pleomórfico, apresentando, inexplicavelmente, metástases regionais ou distantes, ficando na seção dos tumores epiteliais malignos, ao lado do carcinoma ex-adenoma pleomórfico. ${ }^{3,4}$ Em 2017, a OMS reclassificou o tumor como neoplasia epitelial benigna, mantendo advertência para sua natureza. ${ }^{3,4}$ Aproximadamente $6 \%$ de todos os adenomas pleomórficos sofrem transformação maligna para carcinomas ex-adenomas, o que, especula-se, está ligado a instabilidades genéticas do tumor original. ${ }^{3,4}$ Czader propôs que o adenoma pleomórfico metastático está posicionado intermediariamente em um contínuo entre o adenoma pleomórfico e o carcinoma ex-adenoma. ${ }^{3,4}$

O sítio inicial mais comum do APM é a parótida (74\%), seguida das glândulas salivares menores (17\%) e glândulas 
submandibulares $(10 \%) .{ }^{5} \mathrm{O}$ sítio mais comum de metástase é o osso (45\%), seguido por cabeça e pescoço (43\%), pulmões $(36 \%)$ e vísceras abdominais $(10 \%){ }^{5}$

A aparência do adenoma pleomórfico primário, de sua recorrência e de sua metástase são similares. ${ }^{6} \mathrm{Na}$ maioria das situações são massas sólidas sem sinais de hemorragia ou necrose. ${ }^{6}$ As análises histopatológicas dessas neoplasias são similares, sendo evidenciado estroma condromixoide com células fusiformes e mioepiteliais. ${ }^{6}$

O mecanismo de metastização do APM não está claro. Teoriza-se que manipulação cirúrgica determina deslocamento de células tumorais, levando a implantes vasculares, com posterior disseminação metastática. ${ }^{2}$ Esse mecanismo já está bem estabelecido em casos de metastização de outras lesões benignas, como na endometriose - teoria do implante direto, que sugere a ocorrência de implantes peritoneais de células endometriais que escapam através de uma incisão uterina $^{7}$ - e também, em casos de implantes peritoneiais de tecido trofoblástico, após abordagem cirúrgica de gravidez tubária. ${ }^{8}$ Uma rota linfática de disseminação para o adenoma metastático também já foi proposta. ${ }^{2}$ Em comum, a maioria dos pacientes com APM (81\%) apresentava pelo menos uma recorrência local antes da detecção de metástase; entretanto, casos sem evidência de recorrência local têm sido descritos. ${ }^{2,5}$

Por outro lado, análises moleculares evidenciaram diferenças genéticas que explicam o potencial metastático do adenoma pleomórfico, mas ainda não permitiram definições. ${ }^{1}$ Jin et al., em análise cromossomial do adenoma pelomórfico que metastizou para osso, encontrou translocações não-balanceadas que não são encontradas normalmente na variante não-metastática. ${ }^{1}$ Yu identificou aumento da expressão de CD 105, glicoproteína formadora do receptor do fator de crescimento $\beta$ (TGF- $\beta$ ), em vasos tumorais recém formados, tanto em adenomas metastáticos como em carcinomas ex-adenomas. ${ }^{9}$ Já se sabe que a expressão de CD 105 está aumentada em neoplasias de mama, células renais, pâncreas, leucemias e outras, e que, nesses tumores, se relaciona com menores taxas de sobrevida global dos pacientes. ${ }^{9}$

Entretanto, outros trabalhos revelaram semelhanças na expressão de certos oncogenes entre as variantes não metastáticas e a metastática do adenoma pleomórfico. Takeuchi et al. não encontrou alterações na expressão de oncogenes e genes supressores tumorais na variante metastática (bcl2, c-erbB-2, c-myc, L-myc, N-myc, Ha-ras, K-ras) ${ }^{1}$ e nessa

\section{REFERÊNCIAS}

1. Bhutta M, Dunk L, Molyneux A, Tewary A. Parotid pleomorphic adenoma with solitary renal metastasis. Br J Oral Maxillofac Surg. 2010;48(1):61-3.

2. Ghosh A, Dip N, Arundhati M, Asthana A. Pleomorphic adenoma of the parotid gland metastasizing to the scapular region. Acta Cytol. 2008;52 (6):733-5.

3. Watson M, McAllister P, Conn B, MacNeill M, Handley TP. mesma variante, Hoorweg et al. não encontrou diferenças na expressão de proteínas de apoptose (p53, bcl-2, MIB1, CD105, p27, p21). ${ }^{1}$

O tratamento do adenoma pleomórfico, de sua recorrência e das metástases é cirúrgico. ${ }^{6}$ Enucleação do tumor primário nunca deve ser realizada por aumentar a taxa de recorrência local. ${ }^{5}$

A radioterapia persiste como tratamento controverso e tem um papel ainda indefinido na prevenção de recorrências futuras e metástases, bem como no tratamento de sítios inacessíveis cirurgicamente. ${ }^{6}$ Somente a excisão das metástases mostrou benefício na sobrevida. ${ }^{1}$

O APM não pode ser subestimado, pois apresenta comportamento agressivo com uma sobrevida em 5 anos de $58 \%{ }^{1}$ Múltiplas metástases são, invariavelmente, fatais. ${ }^{1}$ Nouraei et al concluíram que os pacientes que apresentaram lesão metastática em 10 anos tiveram um prognóstico significativamente pior em comparação com aqueles que apresentaram metástase após 10 anos da aparição do tumor primário. $^{2}$

Com excisão cirúrgica, felizmente, o prognóstico dos pacientes é geralmente favorável. ${ }^{6} \mathrm{Na}$ série de Wenig et al, $82 \%$ dos pacientes estavam vivos livres de doença ou tinham falecido de causas não relacionadas durante um seguimento de 16 anos depois do diagnóstico. ${ }^{6}$ Nenhum outro estudo forneceu seguimento de longo período com uma quantidade substancial de pacientes, mas isoladamente, publicações sugerem que o prognóstico do APM é bom, apesar de $20 \%$ dos pacientes falecerem devido às metástases. ${ }^{6}$ Foi defendido, então, que a investigação de um adenoma pleomórfico de glândula salivar localmente recorrente deve incluir uma busca por metástases à distância e deve ter um acompanhamento a longo prazo. ${ }^{2}$

\section{CONCLUSÃO}

Adenomas pleomórficos metastáticos são uma entidade rara e controversa, que suscitam debate sobre nomenclatura e critérios diagnósticos. ${ }^{4,6}$ Os pacientes se apresentam após anos do tratamento do seu tumor primário com recorrência local e metástase sem características de malignidade. ${ }^{6}$ Por seu comportamento clínico, o APM, pode-se dizer, está situado na fronteira entre a malignidade e a benignidade. A existência dessa condição, portanto, reitera a necessidade da excisão completa e meticulosa do adenoma pleomórfico primário, bem como do seguimento clínico do paciente. ${ }^{6}$

Metastasising pleomorphic salivary adenoma: a rare case report of a massive untreated minor salivary gland pleomorphic adenoma with concurrent ipsilateral cervical node mestastases. Head Neck Pathol. 2019;13(3):500-6.

4. Knight J, Ratnasingham K. Metastasising pleomorphic adenoma: systematic review. Int J Surg. 2015;19:137-45.

5. Ranjbari N, Panah NH. Benign metastasizing pleomorphic 
adenoma: a case report. Jentashapir J Health Res. 2014;5(6):6-7.

6. Steele NP, Wenig BM, Sessions RB. A case of pleomorphic adenoma of the parotid gland metastasizing to a mediastinal lymph node. Am J Otolaryngol. 2007;28:130-3.

7. Khan Z, Zanfagnin V, El-Nashar SA, Famuyide AO, Daftary GS, Hopkins MR. Risk factors, clinical presentation and outcomes for abdominal wall endometriosis. J Minim Invasive Gynecol. 2017; 24(3):478-84.
8. Ben-Arie A, Goldchmit R, Dgani R, Hazan Y, Ben-Hur H, Open $\mathrm{M}$, et al. Trophoblastic peritoneal implants after laparoscopic treatment of ectopic pregnancy. Eur J Obstet Gynecol Reprod Biol. 2001;96:113-5.

9. Yu M, Li X, Liang R, Yang J, Zhang Y, Wang H. A new ligand of CD105 screened out by phage display technology provides a reliable identification of recurrent or metastasizing pleomorphic adenoma from pleomorphic adenoma. Int Immunopharmacol. 2018;65:47-43.

\section{Como citar:}

Lima GS, Freitas JC, Rolim FJ, Pereira GM, Pereira FJ Filho, Ferreira LA, et al. Metástase linfonodal de adenoma pleomórfico recidivado relato de caso. Rev Med UFC. 2019 jan-mar;60(1):55-58. 